\title{
Fermionic long range correlations realized by particles obeying deformed statistics
}

\author{
Andreas Osterloh ${ }^{1,2}$, Luigi Amico ${ }^{2}$, and Ulrich Eckern ${ }^{1}$ \\ ${ }^{1}$ Institut für Physik, Universität Augsburg, D-86135 Augsburg, Germany \\ ${ }^{2}$ Dipartimento di Metodologie Fisiche e Chimiche (DMFCI), Universitá di Catania, viale A. Doria 6, I-95129 Catania, Italy. \\ Istituto Nazionale per la Fisica della Materia, Unitá di Catania, Italy.
}

\begin{abstract}
Deformed exchange statistics is realized in terms of electronic operators. This is employed to rewrite Hubbard type lattice models for particles obeying deformed statistics (we refer to them as deformed models) as lattice models for electrons. The resulting models show up gauge-like modulations in the hopping processes, which induce long-range correlations in the lattice. The conditions for the Bethe ansatz solvability of the latter are interpreted as restrictions imposed on the statistics to be compatible with the Bethe ansatz solvability of the deformed models. It is found that solvable deformed models are not unitarily equivalent to fermionic models if the exchange of particles with the same spin-orientations is deformed. Statistics deformations, where the exchange relation of two particles is influenced by the presence of other particles, cannot be realized by fermionic operators.
\end{abstract}

The statistics of degrees of freedom drastically affects the physical properties of a many-particle system. Besides bosonic and fermionic statistics, a continuous family of intermediate statistics serves to explain important effects involved in two or one dimensional physics. Remarkably, in $D=2$, excitations in the fractional quantum Hall effect can be described as anyond 1. Onedimensional systems can occur either because only onedimensional dynamics is allowed in the system (even if the system lives in higher dimensions) or because the samples are indeed one-dimensional (like quantum wires, carbon nanotubes, systems with charge density wave order, etc). In the present paper we shall focus on deformed statistics in one dimension.

Defining arbitrary statistics in one dimension exhibits several peculiarities 3 . In particular, imposing the statistics in one dimension can be interpreted as a "continuity condition" on the wave function (arising from the set of coordinates such that two or more particles coincide), fixing its symmetry. One dimensional fractional statistics arises since this constraint on the wave function can be imposed arbitrarily. Explicit realizations of $1 D$ fractional statistics quasi particles, formulated in "first quantization", are the eigenstates of Calogero-Sutherland models.

In Refs. 1 and 5 , the notion of Deformed Exchange Statistics (DES) was defined as a specific deformation of electronic commutation rules (in second quantization). Mathematical aspects of this type of statistics have been also studied in Refs. 6,7. We applied DES to investigate how robust is the solvability by Coordinate Bethe Ansatz (CBA) 9 of the XXZ and the Hubbard model with respect to such a modification of the particle content (we have called those DES preserving CBA solvability Solvable DES).

Following different physics, H. Schulz and B. S. Shastry 10 have included long range correlations in the Hubbard model through a gauge-like modification of the kinetic term of the Hamiltonian. In Ref. 5 we have extended the CBA solution of Schulz-Shastry models to models where more general forms of the correlation have been considered; characterizations focusing on CBA solvability of such kind of correlated models have been reached. The Bethe equations arising from solvable Schulz-Shastry type models show that the long range correlations induce a twist in the boundary conditions.

In the present paper we show that the solvable deformed Hubbard model (without Schulz-Shastry type correlations) is equivalent to adding correlations similar to those discussed by Schulz and Shastry 10 to the undeformed Hubbard model. We prove this realizing DES operators by composites of electronic operators. Using the results of Ref. 11 we can characterize DES which preserve the CBA solvability of the undeformed model (see Eq (17).

Particles obeying DES have creation and annihilation operators obeying the following deformed commutation rules:

$$
\begin{aligned}
& f_{j, \sigma}^{\dagger} f_{k, \sigma^{\prime}}+\mathcal{Q}_{j, k}^{\sigma, \sigma^{\prime}} f_{k, \sigma^{\prime}} f_{j, \sigma}^{\dagger}=\delta_{j, k} \delta_{\sigma \sigma^{\prime}}, \\
& f_{j, \sigma} f_{k, \sigma^{\prime}}+\mathcal{Q}_{k, j}^{\sigma^{\prime}, \sigma} f_{k, \sigma^{\prime}} f_{j, \sigma}=0,
\end{aligned}
$$

Such an algebra is consistent for non trivial $\mathcal{Q}_{j, k}$ if:

$$
\mathcal{Q}_{j, k}^{\sigma \sigma^{\prime}}=\left(\mathcal{Q}_{k, j}^{\sigma^{\prime}, \sigma}\right)^{-1}=\left(\mathcal{Q}_{k, j}^{\sigma^{\prime}, \sigma}\right)^{\dagger}
$$

Furthermore it will be postulated

$$
\left[f_{j, \sigma}^{\dagger}, \mathcal{Q}_{j, k}^{\sigma, \sigma^{\prime}}\right]=\left[f_{j, \sigma}, \mathcal{Q}_{j, k}^{\sigma, \sigma^{\prime}}\right]=0 .
$$

The operators $\nu_{j, \sigma} \doteq f_{j, \sigma}^{\dagger} f_{j, \sigma}$ are the particle-number operators. Note that the relation (11) is formally analog to quon commutation rules (called $q$-CCR in Ref. 6), where relation (2) howezer cannot hold except for the fermionic and bosonic case 12 . Here, in contrast to the quon algebra, the deformation parameter depends on site and spin 
indices $\left(j, \sigma \mid k, \sigma^{\prime}\right)$ (this statistics is of the type called $q_{i j}{ }^{-}$ CCR in Ref. E).

The Equations (3) and (4) guarantee that the particles are representations of the permutation group $S_{N}$ and ensure the validity of the standard commutation relations: $\left[\nu_{i, \sigma}, \nu_{j, \sigma^{\prime}}\right]=0,\left[\nu_{i, \sigma}, f_{j, \sigma^{\prime}}^{\dagger}\right]=\delta_{i, j} \delta_{\sigma, \sigma^{\prime}} f_{j, \sigma^{\prime}}^{\dagger}$, and $\left[\nu_{i, \sigma}, f_{j, \sigma^{\prime}}\right]=-\delta_{i, j} \delta_{\sigma, \sigma^{\prime}} f_{j, \sigma^{\prime}}$. This provides a well defined Fock representation of the algebra defined in Eqs. (11) and (2).

The key observation is that the DES defined by Eqs. (11)-(依) are representable using operators which are composites of electronic creation/annihilation operators.

$$
f_{i, \sigma}^{\dagger}:=c_{i, \sigma}^{\dagger} \exp i\left[\Delta_{i ; l}^{\sigma ; \lambda} n_{l, \lambda}+\Delta_{i ; l, m}^{\sigma ; \lambda, \mu} n_{l, \lambda} n_{m, \mu}+\ldots\right],
$$

where, without loss of generality, $\Delta$ vanishes for any two coinciding index pairs and is symmetric in exchanging arbitrary index pairs behind the semicolon. The number operators remain unchanged after this realization: $\nu$ transport into the number operators $n$ for fermions. The resulting deformation parameters are

$$
\begin{aligned}
\mathcal{Q}_{j, k}^{\sigma, \sigma^{\prime}}= & \exp i\left[\left(\Delta_{k ; j}^{\sigma^{\prime} ; \sigma}-\Delta_{j ; k}^{\sigma ; \sigma^{\prime}}\right)+\right. \\
& \left.+2\left(\Delta_{k ; j, m}^{\sigma^{\prime} ; \sigma, \mu}-\Delta_{j ; k, m}^{\sigma ; \sigma^{\prime}, \mu}\right) n_{m, \mu}+\ldots\right] .
\end{aligned}
$$

From Eq.(16) it is seen that no deformation occurs iff $\Delta$ is totally symmetric in the index pairs. Thus, it will be assumed being antisymmetric in exchanging one index-pair behind the semicolon with the index pair in front of it. This already implies that $\Delta$ vanishes if it has more than two index pairs. The deformation parameter expressed in terms of the parameters $\Delta$ 's in Eq. (5) is

$$
\mathcal{Q}_{j, k}^{\sigma, \sigma^{\prime}}=\exp 2 \mathrm{i} \Delta_{k ; j}^{\sigma^{\prime} ; \sigma}=\exp -2 \mathrm{i} \Delta_{j ; k}^{\sigma ; \sigma^{\prime}} .
$$

As a consequence it is impossible to represent correlated DES (that is DES with $\mathcal{Q}_{j, k}^{\sigma, \sigma^{\prime}}$ being a functional of $n_{m, \mu}$ ) through the realization (5).

Before we continue the discussion of DES, it is worthwhile noting that a totally symmetric part in $\Delta$ creates correlated hopping (without changing the statistics of the particles). For this reason, the fermionic hopping term, which is created by the realization (5) of the degrees of freedom of the deformed Hubbard model is calculated for general $\Delta$ :

$$
H=-t \sum_{i, \sigma}\left(f_{i, \sigma}^{\dagger} f_{i+1, \sigma}+\text { h.c. }\right)+U \sum_{i} \nu_{i, \uparrow} \nu_{i, \downarrow},
$$

where $f_{i, \sigma}, f_{i, \sigma}^{\dagger}(\sigma \in\{\uparrow, \downarrow\}$, or equivalently $\sigma \in$ $\{1 / 2,-1 / 2\}$ ), obey the deformed relations (11) and (2). The two contributions in the Hamiltonian are the hopping term (the $t$-term) and the Coulomb interaction term (the $U$-term). Now we realize the operators $f_{i, \sigma}^{\dagger}$ through electronic operators, using Eq. (5). Then the $t$-term in (8) is rewritten as

$$
\begin{aligned}
& f_{j+1, \sigma}^{\dagger} f_{j, \sigma}=c_{j+1, \sigma}^{\dagger} c_{j, \sigma} \exp i\left[-\Delta_{j+1 ; j}^{\sigma ; \sigma}+\right. \\
& +\left(\Delta_{j+1 ; m}^{\sigma ; \mu}-\Delta_{j ; m}^{\sigma ; \mu}-2 \Delta_{j+1 ; j, m}^{\sigma ; \sigma, \mu}\right) n_{m, \mu}+ \\
& +\left(\Delta_{j+1 ; l, m}^{\sigma ; \lambda, \mu}-\Delta_{j ; l, m}^{\sigma ; \lambda, \mu}-3 \Delta_{j+1 ; j, m, l}^{\sigma ; \sigma, \mu, \lambda}\right) n_{l, \lambda} n_{m, \mu}+ \\
& \quad+\ldots] .
\end{aligned}
$$

Taking explicitly account for the subrelevant parts, i.e. the terms in which the number operator $n_{j, \sigma}$ appears, this is equivalent to

$$
\begin{gathered}
f_{j+1, \sigma}^{\dagger} f_{j, \sigma}=c_{j+1, \sigma}^{\dagger} c_{j, \sigma} \exp i[ \\
\left(\tilde{\Delta}_{j+1 ; m}^{\sigma ; \mu}-\tilde{\Delta}_{j ; m}^{\sigma ; \mu}\right) n_{m, \mu}+ \\
+\left(\tilde{\Delta}_{j+1 ; l, m}^{\sigma ; \lambda, \mu}-\tilde{\Delta}_{j ; l, m}^{\sigma ; \lambda, \mu}\right) n_{l, \lambda} n_{m, \mu}+ \\
+\ldots]
\end{gathered}
$$

where $\tilde{\Delta}$ is the same as $\Delta$ except that $\tilde{\Delta}_{j+1, j, \ldots}^{\sigma ; \sigma, \ldots}=0$ now 11 . We find that the solvability conditions obtained in Ref. 11 are all fulfilled for $\tilde{\Delta}$ and hence the boundary phases are then given by

$$
\sum_{j=1}^{L}\left(\tilde{\Delta}_{j+1, \ldots}^{\sigma, \ldots}-\tilde{\Delta}_{j, \ldots}^{\sigma, \ldots}\right)=0 .
$$

Hence we obtain as result that a totally symmetric part can always be gauged away without residual boundary phase.

Now we continue the study of DES. That means we now restrict ourselves to antisymmetric $\Delta_{m, n}^{\mu ; \nu}$. All parameters with more than two index pairs are zero.

Since the electron number operators coincide with the $\nu$ operators, the $U$-term in (9) coincides with the Hubbard Coulomb interaction: $U \sum_{i} n_{i, \uparrow} n_{i, \downarrow}$. Writing the $t$-term in the form used in the Refs. 10 and 11, namely

$$
\begin{aligned}
\sum_{j, \sigma} & \left\{c_{j+1, \sigma}^{\dagger} c_{j, \sigma} \exp \left(\mathrm{i} \gamma_{j}(\sigma)\right) \times\right. \\
& \left.\times \exp \left[\mathrm{i} \sum_{l}\left(\alpha_{j, l}(\sigma) n_{l,-\sigma}+A_{j, l}(\sigma) n_{l, \sigma}\right)\right]+\text { h.c. }\right\},
\end{aligned}
$$

we can compare with Eq. (9), and the parameters can be identified being

$$
\begin{aligned}
\gamma_{j}(\sigma) & =-\Delta_{j+1 ; j}^{\sigma ; \sigma}, \\
\alpha_{j, m}(\sigma) & =\Delta_{j+1 ; m}^{\sigma ;-\sigma}-\Delta_{j ; m}^{\sigma ;-\sigma}, \\
A_{j, m}(\sigma) & =\Delta_{j+1 ; m}^{\sigma ; \sigma}-\Delta_{j ; m}^{\sigma ; \sigma} .
\end{aligned}
$$

The deformed Hubbard modelis CBA solvable if the following conditions are fulfilled 11 : 


$$
\begin{aligned}
\alpha_{m, j+1}(-\sigma)-\alpha_{m, j}(-\sigma)= & \alpha_{j, m+1}(\sigma)-\alpha_{j, m}(\sigma), \\
A_{m, j+1}(\sigma)-A_{m, j}(\sigma)= & A_{j, m+1}(\sigma)-A_{j, m}(\sigma) \\
& \text { for } m \neq j, j \pm 1 .
\end{aligned}
$$

These conditions for CBA solvability can be expressed directly in terms of the deformation parameters $\mathcal{Q}_{j, k}^{\sigma, \sigma^{\prime}}$ as

$$
\frac{\mathcal{Q}_{j, k+1}^{\sigma, \sigma^{\prime}} \mathcal{Q}_{j+1, k}^{\sigma, \sigma^{\prime}}}{\mathcal{Q}_{j, k}^{\sigma, \sigma^{\prime}} \mathcal{Q}_{j+1, k+1}^{\sigma, \sigma^{\prime}}}=1
$$

for $k \neq j, j \pm 1 \vee \sigma \neq \sigma^{\prime}$.

If the conditions (17) are fulfilled, the Bethe equations for periodic boundary conditions are

$$
\begin{aligned}
& \mathrm{e}^{\mathrm{i} p_{j} L}=\mathrm{e}^{-\mathrm{i} \Phi_{\uparrow}} \prod_{a=1}^{N_{\downarrow}} \frac{\mathrm{i}\left(\sin p_{j}-\zeta_{a}\right)-\frac{U}{4 t}}{\mathrm{i}\left(\sin p_{j}-\zeta_{a}\right)+\frac{U}{4 t}}, \\
& \prod_{\substack{b=1 \\
b \neq a}}^{N_{\downarrow}} \frac{i\left(\zeta_{a}-\zeta_{b}\right)+\frac{U}{2 t}}{i\left(\zeta_{a}-\zeta_{b}\right)-\frac{U}{2 t}}=\mathrm{e}^{-\mathrm{i}\left(\Phi_{\uparrow}-\Phi_{\downarrow}\right)} \prod_{l=1}^{N} \frac{\mathrm{i}\left(\sin p_{l}-\zeta_{a}\right)-\frac{U}{4 t}}{\mathrm{i}\left(\sin p_{l}-\zeta_{a}\right)+\frac{U}{4 t}} .
\end{aligned}
$$

where the boundary twists are given by

$$
\Phi_{\sigma}:=\phi(\sigma)+\phi_{\uparrow \downarrow}^{(1)}(\sigma) N_{-\sigma}+\phi_{\uparrow \uparrow}^{(1)}(\sigma)\left(N_{\sigma}-1\right) .
$$

They can be written in terms of the parameters entering the statistics in the following way:

$$
\begin{aligned}
\phi_{\uparrow \downarrow}^{(1)}(\sigma)= & \sum_{j=1}^{L} \alpha_{j, m}(\sigma)= \\
= & \sum_{j=1}^{L}\left(\Delta_{j+1 ; m}^{\sigma ;-\sigma}-\Delta_{j ; m}^{\sigma ;-\sigma}\right)=0, \\
\phi_{\uparrow \uparrow}^{(1)}(\sigma)= & \sum_{\substack{j=1 \\
j \neq m-1, m}}^{L} A_{j, m}(\sigma)+ \\
& +A_{m, m-1}(\sigma)+A_{m-1, m+1}(\sigma) \\
= & \sum_{j=1}^{L}\left(\Delta_{j+1 ; m}^{\sigma ; \sigma}-\Delta_{j ; m}^{\sigma ; \sigma}\right)+ \\
& +\Delta_{m+1 ; m-1}^{\sigma ; \sigma}-\Delta_{m ; m-1}^{\sigma ; \sigma}+\Delta_{m ; m+1}^{\sigma ; \sigma}- \\
& \Delta_{m-1 ; m+1}^{\sigma ; \sigma}+\Delta_{m-1 ; m}^{\sigma ; \sigma}-\Delta_{m+1 ; m}^{\sigma ; \sigma} \\
= & 2\left(\Delta_{m+1 ; m-1}^{\sigma ; \sigma}+\Delta_{m ; m+1}^{\sigma ; \sigma}+\Delta_{m-1 ; m}^{\sigma ; \sigma}\right), \\
\phi(\sigma)= & \sum_{j=1}^{L}\left(\gamma_{j}(\sigma)+A_{j, j}(\sigma)\right)= \\
= & \sum_{j=1}^{L}\left[-\Delta_{j+1 ; j}^{\sigma ; \sigma}+\Delta_{j+1 ; j}^{\sigma ; \sigma}\right]=0 .
\end{aligned}
$$

Thus the total boundary phase is obtained as
$\Phi_{\sigma}=2\left(\Delta_{m+1 ; m-1}^{\sigma ; \sigma}+\Delta_{m ; m+1}^{\sigma ; \sigma}+\Delta_{m-1 ; m}^{\sigma ; \sigma}\right)\left(N_{\sigma}-1\right)$.

Using Eq. (7) we get

$$
\operatorname{expi} \Phi_{\sigma}=\mathcal{Q}_{m-1, m+1}^{\sigma, \sigma} \mathcal{Q}_{m+1, m}^{\sigma, \sigma} \mathcal{Q}_{m, m-1}^{\sigma, \sigma} .
$$

We point out that the only non-vanishing phases arise from statistics deformation for particles having the same spin orientation.

A consequence of this result is that every translational invariant uncorrelated DES does not affect the spectrum. This includes purely spin dependent DES. This can be seen noting that for translational invariant deformation parameter, i.e. $\mathcal{Q}_{j, k}^{\sigma, \sigma^{\prime}}=: \mathcal{Q}_{j-k}^{\sigma, \sigma^{\prime}}$, the solvability condition (17) must hold without exception 11 . Thus we have

$$
\frac{\mathcal{Q}_{j-k-1}^{\sigma, \sigma^{\prime}} \mathcal{Q}_{j+1-k}^{\sigma, \sigma^{\prime}}}{\mathcal{Q}_{j-k}^{\sigma, \sigma^{\prime}} \mathcal{Q}_{j-k}^{\sigma, \sigma^{\prime}}} \stackrel{!}{=} 1
$$

for arbitrary $j, k, \sigma$ and $\sigma^{\prime}$. For $\sigma=\sigma^{\prime}$ and $j=k+1$ this yields

$$
\frac{\mathcal{Q}_{0}^{\sigma, \sigma} \mathcal{Q}_{2}^{\sigma, \sigma}}{\mathcal{Q}_{1}^{\sigma, \sigma} \mathcal{Q}_{1}^{\sigma, \sigma}}=\mathcal{Q}_{2}^{\sigma, \sigma} \mathcal{Q}_{-1}^{\sigma, \sigma} \mathcal{Q}_{-1}^{\sigma, \sigma}=1
$$

which is exactly $\exp -\mathrm{i} \Phi_{\sigma}$ in the r.h.s. of Eq. (24) for the translational invariant case. For deformations of the statistics which are not translational invariant, the spectrum is modified even for a free gas of such particles instead. In the limit $U \longrightarrow 0$, the phases have to be picked up by proper convergence of $\sin p_{j}$ and $\zeta_{a}$ linearly in $U / t$. As a result, $N_{\uparrow}$ momenta differ from the undeformed values $p_{j}^{0}=\frac{2 \pi}{L} k$ by $\left(\Phi_{\uparrow} \bmod 2 \pi\right) / L$ and $N_{\downarrow}$ momenta differ by $\Phi_{\downarrow} / L$. The energy formula has the form

$$
E=-2 t \sum_{\sigma} \sum_{i_{\sigma}=1}^{N_{\sigma}} \cos \left(\frac{2 \pi}{L} l_{i_{\sigma}}+\frac{\Phi_{\sigma}}{L}\right) .
$$

For finding the ground state, one has to find the distribution of the distinct integers $l_{i_{\sigma}}$ leading to minimal energy. This distribution depends on the filling, discriminating less than quarter filling from fillings in between $1 / 4$ and $1 / 2$. Fillings beyond half filling are to be extracted from the Bethe result explpiting the particle-hole symmetry of the Hubbard model13. In the thermodynamic limit, due to the absent symmetry of the momentum distribution for the ground state, a finite contribution of the integral over the momenta to order $(1 / L)^{0}$ comes out besides finite size corrections to order $(1 / L)^{1}$. Since the energy itself is of order $L$, we will turn to energy densities, i. e. the energy per site $\varepsilon:=\frac{E}{L}$. Defining $N_{\min }:=\min \left(N_{\uparrow}, N_{\downarrow}\right), \epsilon_{\sigma}:=\Phi_{\sigma} \bmod 2 \pi$, 
$\epsilon_{c m}:=\left(\epsilon_{\uparrow}+\epsilon_{\downarrow}\right) / 2$, and $\Delta \epsilon:=\left|\epsilon_{\uparrow}-\epsilon_{\downarrow}\right| / 2$ one obtains for the ground state energy density $\varepsilon_{\Phi}^{0}=: \varepsilon_{0}^{0}+\Delta \varepsilon^{0}$

$$
\Delta \varepsilon^{0}= \begin{cases}-\frac{2 t}{\pi L} \Delta \epsilon\left(1-\cos \frac{2 \pi N_{\min }}{L}\right)+ & \\ +\frac{t}{\pi L^{2}}\left(\epsilon_{c m}+\Delta \epsilon\right)^{2} \sin \frac{\pi N}{L}- & ; \Phi_{\uparrow} \Phi_{\downarrow}<0, \\ -\frac{2 t}{\pi L^{2}} \epsilon_{c m} \Delta \epsilon \sin \frac{\pi\left|N_{\uparrow}-N_{\downarrow}\right|}{L} & \\ -\frac{2 t}{\pi L} \Delta \epsilon\left(\cos \frac{\pi N}{L}+\cos \frac{\pi\left|N_{\uparrow}-N_{\downarrow}\right|}{L}\right)+ & \\ +\frac{t}{\pi L^{2}}\left(\epsilon_{c m}+\Delta \epsilon\right)^{2} \sin \frac{\pi N}{L}- & ; \Phi_{\uparrow} \Phi_{\downarrow}>0 \\ -\frac{2 t}{\pi L^{2}}\left(\epsilon_{c m}^{2}+\Delta \epsilon^{2}\right) \sin \frac{\pi\left|N_{\uparrow}-N_{\downarrow}\right|}{L} & \frac{1}{4} \leq \frac{N}{2 L} \leq \frac{1}{2} \\ -\frac{2 t}{\pi L} \Delta \epsilon\left(\cos \frac{\pi N}{L}-\cos \frac{\pi\left|N_{\uparrow}-N_{\downarrow}\right|}{L}\right)+ & \\ +\frac{t}{\pi L^{2}}\left(\epsilon_{c m}+\Delta \epsilon\right)^{2} \sin \frac{\pi N}{L}- & ; \Phi_{\uparrow} \Phi_{\downarrow}>0 \\ -\frac{2 t}{\pi L^{2}}\left(\epsilon_{c m}^{2}+\Delta \epsilon^{2}\right) \sin \frac{\pi\left|N_{\uparrow}-N_{\downarrow}\right|}{L} & \frac{N}{2 L}<\frac{1}{4}\end{cases}
$$

It is seen that a contribution linear in $1 / L$ can occur only for non-vanishing $\Delta \epsilon$. Otherwise, the effect is only visible in finite systems to second order in $1 / L$. On the other hand $\Delta \epsilon=0$ also implies that there is no phase factor in front of the spin part of the Bethe equation, so that an application of the string hypothesis is unaffected for this case.

In conclusion, we have shown how DES is connected to long range correlations of Schulz-Shastry type. The connection has been revealed realizing operators obeying DES through composites of fermionic operators. From Eqs. (18) - 23) we conclude that solvable DES produces modifications in the boundary condition (supporting the general idea of the relationship between statistics and topology in $1 D)$. It is important noticing that this is a peculiarity of solvable DES. DES which are not solvable affect the physics in a different (more complicated) way. Preliminary results indeed have shown that they modify the spectrum of a system with open boundary conditions. The characterization concerning the CBA solvability of the Schulz-Shastry type models 11 provides the conditions, which the deformation $\mathcal{Q}$ must fulfill in order that the deformed Hubbard model is CBA solvable. The results obtained in Refs. 4,5 appear as special cases within the class of statistics studied here. It is worthwhile noting that deformation effects can be seen already for a free gas of such particles. Its ground state energy as a function of the deformation parameters is calculated up to second order in $1 / L$, and it contains also a contribution linear in $1 / L$ and in the asymmetry in the boundary phases $\Delta \varepsilon$.

We found that correlated deformed exchange statistics (with $\mathcal{Q}_{j, k}^{\sigma, \sigma^{\prime}}$ being a functional of $n_{m, \mu}$ ) cannot be real- ized in terms of fermionic operators. Work is in progress along this directions.

\section{ACKNOWLEDGMENTS}

Motivating discussions with G. Falci, R. Fazio, G. Giaquinta, A. Kundu, M. Rasetti, and P. Schwab are gratefully acknowledged besides the support through the SFB 484 and the Graduiertenkolleg "Nonlinear Problems in Analysis, Geometry, and Physics" (GRK 283), financed by the German Science Foundation (DFG) and the State of Bavaria.

${ }^{1}$ B.I. Halperin, Phys. Rev. B 252185 (1985); A. MacDonald, Phys. Rev. Lett. 64 (1990) 220.

${ }^{2}$ J. Myrheim, Anyons in: Topological Aspects Of Low Dimensional Systems (EDP, Springer, 1998).

${ }^{3}$ A. P. Polychronakos, Generalized statistics in one dimension Topological Aspects Of Low Dimensional Systems (EDP, Springer, 1998).

${ }^{4}$ L. Amico, A. Osterloh, and U. Eckern Phys. Rev. B 58, 1703R (1998) and references therein.

${ }^{5}$ A. Osterloh, L. Amico, and U. Eckern, J. Phys. A 33, L87 (2000).

${ }^{6}$ M. Bozejko and Speicher, Math. Ann. 300, 97 (1994); P.E.T. Jorgensen, L.M. Schmitt, and R,F. Werner, J. Funct. Anal. 134, 33 (1995); P.E.T. Jorgensen, D.P. Proskurin, and Yu.S. Samoilenko, math-ph 0001011.

${ }^{7}$ W. Marcinek, Rep. Math. Phys. 41, 155 (1998).

${ }^{8}$ H. Bethe, Z. Phys. 71 (1931) 205.

${ }^{9}$ B. Sutherland, in Exactly Solvable Problems in Condensed Matter and Relativistic Field Theory, Lecture Notes in Physics 242 eds. B. S. Shastry, S. S. Jha, and V. Singh, (Springer Berlin, 1985).

${ }^{10}$ H.J. Schulz and B.S. Shastry, Phys. Rev. Lett. 80 (1998) 1924.

11 A. Osterloh, L. Amico, and U. Eckern, Nucl. Phys. B 588, 531 (2000).

12 O. W. Greenberg, Phys. Rev. Lett. 64, 705 (1990); Phys. Rev. D 43, 4111 (1991); L.-A. Wu, Z.-Y. Wu, and J. Sun, Phys. Lett. A 170, 280 (1992).

13 E.H. Lieb and F.Y. Wu, Phys. Rev. Lett. 20 (1968) 1445. 